Characterising the meningococcal glycointeractome: what's new?

Kate L. Seib ${ }^{1 *}$, Michael P. Jennings ${ }^{1}$ and Christopher J. Day ${ }^{1 * *}$

${ }^{1}$ Institute for Glycomics, Griffith University, Gold Coast, Queensland, Australia

* Author for correspondence: k.seib@griffith.edu.au

** Author for correspondence: c.day@griffith.edu.au

Photo (headshot) of authors: attached

Keywords: Neisseria meningitidis, glycan, carbohydrate, lectin, host-pathogen interactions, virulence, lipooligosaccharide, capsule, glycoprotein 


\section{Body of article}

Neisseria meningitidis is a Gram-negative bacterium that asymptomatically colonises the upper respiratory tract of approximately $10-20 \%$ of the population. However, it can also cause life threatening invasive meningococcal disease that typically presents as meningitis or septicaemia [1]. Vaccines are available to prevent disease caused by five of the six main meningococcal serogroups (serogroups A, B, C, W, Y) and the bacteria is susceptible to antibiotics [2]. Regardless, there is still a high burden of disease, with an estimated 1.2 million cases and 135,000 deaths each year worldwide. This is likely due to several factors, including the incomplete uptake of available vaccines, the absence of a serogroup $X$ vaccine, and the fact that invasive infection is often difficult to diagnose at early stages and can rapidly progress to a life-threatening disease before antibiotics can be administered. As such, there is an ongoing need for a better understanding of meningococcal pathogenesis to aid development of improved therapeutics and vaccines.

Carbohydrates (also called glycans, oligo/polysaccharides or sugars) have long been known to play an important role in host-pathogen interactions [3]. With respect to N. meningitidis, there are several glycoconjugates expressed of its surface; it has a polysaccharide capsule, a lipooligosaccharide (LOS), and glycosylated outer membrane proteins, including type IV fimbriae (pili) and a surface expressed nitrite reductase, called AniA [reviewed in 4, 5]. On the host side, human microenvironments encountered by $N$. meningitidis have a complex and extensive glycocalyx [6,7] and association with host glycans has been reported in several studies of meningococcal virulence factors [reviewed in 4,5]. These interactions include the serogroup B vaccine antigen NHBA that binds to heparin [8] and heparan sulfate proteoglycans (HSPGs) [9]. These interactions contribute to serum survival and adherence to epithelial cells, respectively. Another key example of glycan targeting by Neisserial adhesins are the opacity proteins, Opc and Opa, which have been reported to bind heparin, HSPGs, galactose, sialic acids, and the extracellular matrix glycoproteins fibronectin and vitronectin [reviewed in 4, 5].

Glycan array technology was recently used as a systematic approach to identify interactions between $N$. meningitidis and host glycans and thereby define the meningococcal glycointeractome. The serogroup B wild-type strain MC58 is reported to bind to 223 of the 367 glycan structures on the glycan array, including blood group antigens, mucins, gangliosides and glycosaminoglycans (GAGs) [10]. Of the interactions revealed in this study, only approximately $30 \%$ of the binding events can be explained by previously known meningococcal lectin activity. Newly identified interactions include binding to mannosyl structures, complex galactose (Gal) structures and hyaluronan GAGs. Using strains lacking expression of key outer membrane structures (i.e., pili, Opc and capsule mutants, and LOS variants), further glycan array studies were performed to determine the meningococcal surface factors responsible for the observed binding to host glycans. In addition, surface plasmon resonance (SPR) and isothermal calorimetry (ITC) analysis was performed with isolated versions of the same set of bacterial structures to confirm binding, and to determine the kinetics of the interactions.

Recent evidence suggests that high affinity glycan-glycan interactions may be a common mechanism of bacterial adherence to host cells [11]. To determine whether glycan-glycan interactions occur during $N$. meningitidis-host cell contact, the major surface glyconjugates, polysaccharide capsule and LOS, were investigated. The structures of the polysaccharide capsules expressed by $N$. meningitidis strains is the basis for serogroup classification, and is the immunising antigen in the serogroup A, C, W and Y vaccines. The serogroup B capsular polysaccharide $((\alpha 2-8)-$ linked polysialic acid) is similar to host glycans (e.g. neural cell adhesion molecule (NCAM)), and is therefore poorly immunogenic and has not been used as a vaccine antigen. Capsule provides the meningococcus with protection from innate and adaptive immune responses and is crucial for invasive disease [1]. Glycan array analysis showed that binding to 40 glycans was lost in a capsule knockout strain $\mathbb{C} 3$ [12], relative to the serogroup B MC58 wild-type strain, suggesting that the 
capsule may directly mediate binding to several glycans [10]. Furthermore, the $\varnothing 3$ acapsulate strain bound 43 additional structures relative to the capsulate wild-type strain, suggesting that some interactions are blocked by the presence of the dense high molecular weight polyanionic polysaccharide coating the bacterial surface. While both the wild-type and acapsulate strain bound glycans from all classes on the array, they differed in their ability to recognise blood group antigens. The acapsulate strain bound to blood group A antigen structures, while the capsulate wildtype strain bound to the $\mathrm{B}$ and $\mathrm{H}$ blood group antigens, indicating variability in blood type preference of acapsulate versus capsulate strains. Blood group antigens are widely distributed in the human body, and are among the most common terminal glycans found on red blood cells, airway epithelial cells and mucins. As such, there have been numerous studies investigating whether blood type or blood antigen secretion status can affect the clinical outcome or susceptibility to pathogenic bacteria and viruses [13]. These new findings may indicate a novel role of host blood group status in the carriage of either capsulate or acapsulate $N$. meningitidis.

The LOS of $N$. meningitidis is made up of lipid A, a core oligosaccharide (containing 3-deoxy-Dmanno-oct-2-ulosonic acid and heptose residues), and variable short oligosaccharides chains. The differences in the composition of these oligosaccharides form the basis of meningococcal immunotyping (L1-L12) and results from the phase variable expression of several glycosyltransferases [14]. Glycan binding by two natural LOS phase variants was investigated, the L3 LOS and the truncated L8 LOS, which lacks the terminal lacto- $N$-neotetraose structure of L3 LOS due to the phase variation of the LgtA glycosyltransferase [14]. N. meningitidis strains expressing either the L8 or L3 immunotype LOS bound a broad range of glycans on the array with a total of 208 glycans common between the strains. Purified LOS structures were used to better characterise the direct binding of the LOS to glycan structures on the array. Glycans bound by L3 and L8 LOS were almost mutually exclusive. The GAG hyaluronan, which is widely distributed in the human body, was bound by both L3 and L8 LOS. This indicates that the LOS of N. meningitidis regardless of immunotype is one of the surface structures responsible for the hyaluronan binding noted in the initial MC58 wild-type array screen. L3 LOS bound short-chain host glycans displaying a terminal galactose, e.g. lacto- $N$-biose I, the Thomsen-Friedenreich (TF) antigen, and large chain fucosylated glycans containing an $N$-acetyllactosamine (Galß1-4GlcNAc) epitope, including lacto- $N$-fucopentaose III, a Lewis X pentasaccharide. L3 LOS also bound a $\alpha 2-8$ linked sialic acid trisaccharide and the GAG heparin. SPR analysis revealed that the interaction between L3 LOS and the TF antigen is the highest affinity glycan-glycan interaction reported to date $\left(\mathrm{K}_{\mathrm{D}}=\right.$ $13 \mathrm{nM}$ ). TF antigen is the most common mucin O-glycan core (core 1) structure and is found on most cell types [3]. Unlike L3 LOS, purified L8 LOS did not bind any glycans with terminal GlcNAc or glucose, and recognised mostly GAGs and sialylated glycans including colominic acid (( $\alpha 2-8)$-linked polysialic acid), an analogue of the $N$. meningitidis serogroup B capsular polysaccharide. These direct glycan-glycan interactions suggest distinct roles for meningococcal immunotypes with respect to host-pathogen interactions and intra-meningococcal interactions.

To further investigate protein-glycan interactions during $N$. meningitidis-host cell contact, a comparison of the glycan binding profile of strains with and without Opc expression (strains $\mathbb{C} 2$ and C9, respectively [12]) was performed. This revealed a loss of binding to 43 glycans in the absence of Opc, which included terminal Gal structures, sialylated glycans and GAGs. ITC analysis revealed that several of these interactions were high affinity (with a dissociation constant $\left(\mathrm{K}_{\mathrm{D}}\right)$ below $5 \mu \mathrm{M})$. Opc bound 2,6 sialylactose $\left(\mathrm{K}_{\mathrm{D}}\right.$ of $\left.\sim 710 \mathrm{nM}\right)$ and sialyl LewisX $\left(\mathrm{K}_{\mathrm{D}}\right.$ of $\left.\sim 590 \mathrm{nM}\right)$ with a affinities similar to the previously observed for these interactions [15]. In addition, novel glycan binding by Opc was observed to chondroitin-6-sulfate polymer $\left(\mathrm{K}_{\mathrm{D}} \sim 630 \mathrm{nM}\right)$ and lacto- $N$ neotetraose $\left(\mathrm{K}_{\mathrm{D}} \sim 1.7 \mu \mathrm{M}\right)$ [10]. Chondroitin-6-sulfate is found in all human extra cellular matrix, and is the major GAG expressed within the central nervous system (CNS) [16], suggesting the potential for Opc-mediated meningococcal adherence to cells within the CNS during meningitis. Lacto- $N$-neotetraose is an integral component of glycosphingolipids such as paragloboside, and is 
also the precursor of the $\mathrm{ABO}$ and $\mathrm{P} 1$ blood group antigens. As mentioned above, $\mathrm{ABO}$ Blood group antigens are found on epithelial cells and mucins and interactions with these glycans may be important for nasopharyngeal colonization.

Pili are adhesins, and in Neisseria pilin glycosylation enhances adherence to epithelial cells and endothelial cells [17], and is required for platelet activating factor receptor-mediated adherence to human airway epithelial cells [18]. To investigate potential pili-based glycointeractions, two pili mutants were examined in a MC58 wild-type and capsule mutant background. Over 50 interactions were lost when comparing the pili mutant strain with the isogenic wild-type strain, regardless of the presence or absence of capsule. The ability of capsule to suppress meningococcal interactions with the host cells described above, and is thought to be due to the polyanionic nature of the polysaccharide. Capsule expression is crucial for meningococcal survival during invasive disease, while the non-encapsulated state is favoured during colonisation [1]. The observed variation in glycan binding by the different pili mutants suggests flexibility in the roles that pili play in these interactions, depending on disease stage. Interestingly, the non-encapsulated pili mutant lost binding to asialo GM1 and GalNAc $\beta 1-4 \mathrm{Gal}$, implying that meningococcal pili mediate these interactions. Asialo GM1 is highly expressed in regenerating respiratory epithelia and is a potential target for meningococcal adherence to these cells. Several other pathogens that colonise the respiratory tract are known to target asialo GM1 (or -GM2), and these interactions are also mediated by pili of Pseudomonas aeruginosa [19].

As the field of glycobiology has expanded, it has become apparent that the human glycome is extensive and is an important target for interactions with various bacterial pathogens. Recent analysis has revealed that $N$. meningitidis binds host glycans from many different structural and functional classes [10], highlighting the diverse glycointeractions that may occur during meningococcal colonisation and disease. The meningococcal proteins and/or glycans that are responsible for all the newly identified glycan interactions are currently under further investigation, and could potentially be targeted for the development of novel antibiotics or vaccines to prevent meningococcal disease.

\section{Financial \& competing interest disclosure}

The authors are supported by the Australian National Health and Medical Research Council [Career Development Fellowship 1045235 to K.L.S., Program Grant 1071659 to M.P.J., Project Grant 1099278 to K.L.S. and C.J.D, and Project Grant 1108124 to M.P.J and C.J.D.].

The authors have no other relevant affiliations or financial involvement with any organization or entity with a financial interest in or financial conflict with the subject matter or materials discussed in the manuscript apart from those disclosed. No writing assistance was utilized in the production of this manuscript.

\section{References}

1. Rouphael NG, Stephens DS. Neisseria meningitidis: Biology, Microbiology, and Epidemiology. Methods in molecular biology (Clifton, N.J.) 799 1-20 (2012).

2. Borrow R, Alarcón P, Carlos J et al. The Global Meningococcal Initiative: global epidemiology, the impact of vaccines on meningococcal disease and the importance of herd protection. Expert Review of Vaccines 16(4), 313-328 (2017).

3. Esko JD, Sharon N. Microbial Lectins: Hemagglutinins, Adhesins, and Toxins. In: Essentials of Glycobiology, Varki A, Cummings RD, Esko JD et al. (Eds). Cold Spring Harbor Laboratory Press, Cold Spring Harbor (NY) (2009). 
4. Mubaiwa TD, Semchenko EA, Hartley-Tassell LE, Day CJ, Jennings MP, Seib KL. The sweet side of the pathogenic Neisseria: the role of glycan interactions in colonisation and disease. Pathogens and disease 75(5), ftx063 (2017).

5. Hill DJ, Griffiths NJ, Borodina E, Virji M. Cellular and molecular biology of Neisseria meningitidis colonization and invasive disease. Clin Sci (Lond) 118(9), 547-564 (2010).

6. Varki A, Esko JD, Colley KJ. Cellular Organization of Glycosylation. In: Essentials of Glycobiology, Varki A, Cummings RD, Esko JD et al. (Eds). Cold Spring Harbor Laboratory Press, Cold Spring Harbor (NY) (2009).

7. Kamhi E, Joo EJ, Dordick JS, Linhardt RJ. Glycosaminoglycans in infectious disease. Biological Reviews 88(4), 928-943 (2013).

8. Serruto D, Spadafina T, Ciucchi L et al. Neisseria meningitidis GNA2132, a heparinbinding protein that induces protective immunity in humans. PNAS 107(8), 3770-3775 (2010).

9. Vacca I, Del Tordello E, Gasperini G et al. Neisserial Heparin Binding Antigen (NHBA) contributes to the adhesion of Neisseria meningitidis to human epithelial cells. PLOS ONE 11(10), (2016).

10. Mubaiwa TD, Hartley-Tassell LE, Semchenko EA et al. The glycointeractome of serogroup B Neisseria meningitidis strain MC58. Scientific Reports 7(1), 5693 (2017).

11. Day CJ, Tran EN, Semchenko EA et al. Glycan:glycan interactions: High affinity biomolecular interactions that can mediate binding of pathogenic bacteria to host cells. PNAS 112(52), E7266-E7275 (2015).

12. Virji M, Makepeace K, Peak IR, Ferguson DJ, Jennings MP, Moxon ER. Opc- and pilusdependent interactions of meningococci with human endothelial cells: molecular mechanisms and modulation by surface polysaccharides. Molecular microbiology 18(4), 741-754 (1995).

13. Cooling L. Blood Groups in Infection and Host Susceptibility. Clinical microbiology reviews $28(3), 801-870$ (2015).

14. Jennings MP, Srikhanta YN, Moxon ER et al. The genetic basis of the phase variation repertoire of lipopolysaccharide immunotypes in Neisseria meningitidis. Microbiology 145(11), 3013-3021 (1999).

15. Moore J, Bailey SES, Benmechernene Z et al. Recognition of Saccharides by the OpcA, OpaD, and OpaB Outer Membrane Proteins from Neisseria meningitidis. Journal of Biological Chemistry 280(36), 31489-31497 (2005).

16. Kwok JCF, Warren P, Fawcett JW. Chondroitin sulfate: A key molecule in the brain matrix. The International Journal of Biochemistry \& Cell Biology 44(4), 582-586 (2012).

17. Virji M, Saunders JR, Sims G, Makepeace K, Maskell D, Ferguson DJP. Pilus-facilitated adherence of Neisseria meningitidis to human epithelial and endothelial cells: modulation of adherence phenotype occurs concurrently with changes in primary amino acid sequence and the glycosylation status of pilin. Molecular Microbiology 10(5), 1013-1028 (1993).

18. Jen FEC, Warren MJ, Schulz BL et al. Dual pili post-translational modifications synergize to mediate meningococcal adherence to platelet activating factor receptor on human airway cells. PLoS Pathogens 9(5), e1003377 (2013).

19. Gupta SK, Berk RS, Masinick S, Hazlett LD. Pili and lipopolysaccharide of Pseudomonas aeruginosa bind to the glycolipid asialo GM1. Infection and Immunity 62(10), 4572-4579 (1994). 